Available online on 15.07.2020 at http://ujpr.org
Universal Journal of Pharmaceutical Research
An International Peer Reviewed Journal
Open access to Pharmaceutical research

\title{
THE RISKS AND ADVANTAGES OF ANTI-DIABETES THERAPY IN THE POSITIVE COVID-19 PATIENT

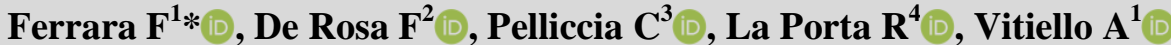 \\ ${ }^{1}$ Usl Umbria 1, Perugia, Italy; ${ }^{2}$ Regione Campania, Naples, Italy. ${ }^{3}$ Usl Umbria 2, Terni, Italy; ${ }^{4}$ Asur Marche, Macerata, Italy
}

\section{ABSTRACT}

The new SARS-Cov-2 (COVID-19) is causing thousands of deaths worldwide and has caused a global pandemic, one of the biggest health challenges ever faced in history. In the most severe cases, SARS-Cov-2 infection can cause fatal lung injuries. In this context, it is essential to recognise effective therapeutic agents against the virus. There are currently no direct and effective vaccines and antivirals available. People with pre-existing conditions, such as diabetes, and with chronic drug therapies in place may represent complex patients difficult to manage clinically during COVID-19 infection and at high risk of major complications. The regulation of blood glucose and the adoption of appropriate measures are critical aspects to consider for the diabetic patient in this pandemic period, especially in the patient with ongoing infection. In this article we describe the current evidence in the literature on the possible risks of side effects caused by taking antidiabetic drugs in the COVID-19 patient and the data on extra homeostasis glycemic activity useful to fight viral infection.

Keywords: COVID-19, diabetes, hyperglycemia, inflammatory, immunomodulants, SARS-Cov-2.

Article Info: Received 25 April 2020; Revised 8 June; Accepted 3 July, Available online 15 July 2020

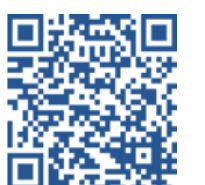

Cite this article-

Ferrara F, De Rosa F, Pelliccia C, La Porta R, Vitiello A. The risks and advantages of anti-diabetes therapy in the positive COVID-19 patient. Universal Journal of Pharmaceutical Research 2020; 5(3):65-67.

DOI: https://doi.org/10.22270/ujpr.v5i3.419

Address for Correspondence

Francesco Ferrara, Farmacista Dirigente, Distribuzione Diretta Area Sud, Usl Umbria 1, Perugia, Italy. E-mail: francesco.ferrara@uslumbrial.it

\section{INTRODUCTION}

The viral infection caused by the new SARS-Cov-2 (COVID-19), recorded its first cases in China at the end of 2019, and within a few months it became a global pandemic. At the time of writing this report, there were about 4.71 million infected cases and 315,000 deaths with over 250 countries affected ${ }^{1}$. COVID-19 infection can occur in several stages, the first with mild or asymptomatic symptoms and the second and third stages more severe, consisting of a generalized hyperactive inflammatory state caused by a cytokinic storm, which can cause fatal lung injury and multi-organ dysfunction that can quickly lead to death. Patients with pre-existing chronic diseases such as diabetes are at high risk of infection and increased complications, and the management of polypharmacy is complex, consisting of therapeutic agents that must treat the disease already present and agents against COVID-19 infection. To date, there are no direct antivirals and effective vaccines against SARS-Cov- $2^{2}$. Clinical pharmacological aspects of the diabetic patient during the COVID-19 infection
The risk of anti-diabetes agents during SARS-Cov-2 infection. Continuous control and monitoring of blood glucose is of paramount importance for the diabetic patient, even more so during an ongoing COVID-19 infection. Currently, guidelines recommend diabetic patients to continue taking routine antidiabetic therapies, and to date there is no evidence that any therapeutic agent against diabetes can increase the risk of COVID-19 infection. Although the patient with diabetes is affected by SARS-Cov-2, blood glucose levels should continue to be monitored and blood glucose levels controlled with drug therapy. In a COVID-19 patient with SARS-Cov-2 infection, and especially in more severe cases, a possible change in dosage, a therapeutic change, or even a discontinuation of the antidiabetes drug could be considered, but one should always consider the case individually depending on the clinical conditions. The most important factors to consider are glycemic normalization and the risk of hypoglycemia/hyperglycemia and the interactions between antidiabetic drugs and drugs used to fight COVID-19 infection. Diabetes therapy in a patient with COVID-19 infection could pose a serious risk of 
causing serious complications if poorly managed. Metformin is among the first-line drugs for type 2 diabetes and is predominantly eliminated in the kidney, in a patient with COVID-19 renal function should be closely monitored to avoid the risk of lactic acidosis caused by increased drug concentration ${ }^{3}$. In addition, some antivirals used to fight COVID-19 are inhibitors of organic cation carrier OCT, metformin is a substrate of this protein, inhibiting OCT the concentrations of metformin in plasma may be higher with risk of lactic acidosis. Peptide-1 glucagon receptor agonist therapy (GLP-1RA) should probably be discontinued in patients with severe hemodynamic instability (compromising absorption from subcutaneous sites), renal dysfunction and gastrointestinal dysfunction. GLP-1RA may cause a delay in gastric emptying that leads to significant reductions in the rate and degree of absorption of certain oral drugs administered simultaneously such as darunavir or remdesivir antivirals ${ }^{4}$. Common side effects of GLP-1RA include nausea, vomiting and diarrhoea, which in a COVID-19 patient with similar gastrointestinal symptoms may lead to loss of electrolytes and risk of cardiac arrhythmias.

Dipeptidil peptidase-4 (DPP4) inhibitors are associated with a low risk of hypoglycemia and are relatively safe in a wide range of renal function, probably this class of drugs can be considered the safest in a COVID-19 patient ${ }^{5}$. The use of SGLT-2 inhibitors is more complex in COVID-19 patients due to the risk of diabetic ketoacidosis, which can lead to potentially fatal cases ${ }^{6}$. The clinical condition of the patient, high concentration of ketone bodies, renal dysfunction are aspects to be considered for a possible interruption of treatment with SGLT-2. In patients with COVID-19 on sulphoniluree therapy, a change in insulin therapy should be considered due to the risk of high hypoglycaemia in patients with SARS-Cov-2 infection. Caution with sulfonylureas should also be exercised in consideration of chloroquine, due to the risk of hypoglycaemia with both and if renal dysfunction is present $^{7}$. The use of thiazolidinediones should be carefully evaluated in a hemodynamically unstable patient with liver dysfunction due to the risk of water retention and edema. Insulin use probably requires dose modification in a COVID-19 patient, the most serious risk is hypoglycemia, but is probably preferable to the use of sulfonylureas and thiazolidinediones.

The advantages of diabetes agents against SARSCOV-2 infection

Normalization of glycemic homeostasis should be carefully performed with routine therapeutic agents, even during the COVID-19 infection phase. However, some evidence has shown that some antidiabetic drugs in COVID-19-like epidemics, such as SARS-COV and MERS, have been effective in combating viral infection. It is now known that in the most severe stages of COVID-19 infection an overactive and uncontrolled inflammatory system caused by a cytokinic cascade is responsible for multi-organ dysfunction and fatal lung lesions. In particular, the use of metformin has shown a decrease in inflammatory markers in patients with SARS-COV and MERS, however there is no clinical data to support this and its use in patients with severe COVID-19 should be carefully assessed in light of the risks described above ${ }^{8,9}$. The use of DPP IV inhibitors is being investigated in patients with COVID-19. The DPP4 protein is expressed in many cells including alveolar epithelium and inflammatory cells. MERS-CoV uses DPP4 to enter host cells ${ }^{10}$, but it is not yet known whether SARS-Cov-2 uses the same vector to enter the cell as ACE-2. If this is demonstrated, the use of DPP IV inhibitors could reduce the risk of Sars-Cov-2 infection, but so far this has not been demonstrated and there are no clinical data to support it $^{11}$. Similarly to metformin, the effects of reducing inflammatory markers are also known for GLP-1RA and have also demonstrated potential therapeutic benefit in patients with diabetes and acute lung injury ${ }^{12}$. However, even in this case the available data are limited to experimental models and their benefit must be demonstrated by clinical data.

\section{CONCLUSION}

The COVID-19 pandemic is causing numerous deaths worldwide and there are currently no vaccines and antivirals directed against the virus. The patient with diabetes and with SARS-Cov-2 infection is a complex patient who needs to be carefully managed clinically, the therapy for diabetes cannot be suspended and glycemic normalization must always be sought, however during the infection phase some common antidiabetes drugs could represent serious risk factors for complications if used incorrectly, for some of them there is also evidence of positive efficacy in the COVID-19 positive patient. Clinical data from wellstructured epidemiological studies will provide the necessary evidence.

\section{AUTHOR'S CONTRIBUTION}

All authors have worked equally for this work.

\section{ACKNOWLEDGEMENTS}

The authors extend their thanks and appreciation to the Regione Campania to provide necessary facilities for this work.

\section{CONFLICT OF INTEREST}

No conflict of interest associated with this work.

\section{REFERENCES}

1. World Health Organization. Coronavirus disease 2019 (COVID-19) situation report.

https://www.who.int/emergencies/diseases/novelcoronavirus-2019/situation-reports

2. Lin L, Lu L, Cao W, Li T. Hypothesis for potential pathogenesis of SARS-Cov-2 infection a review of immune changes in patients with viral pneumonia. Emerg Microbes Infect 2020; 9(1):727-732. https://doi.org/10.1080/22221751.2020.1746199

3. Ralph De Fronzo. Metformin-associated lactic acidosis: Current perspectives on causes and risk Metabolism. 2016; 65, 2, 20-29. https://doi.org/10.1016/j.metabol.2015.10.014

4. Liu J, Li L, Deng K, Xu C, Busse JW, Vandvik PO, et al. Incretin based treatments and mortality in patients with type 2 diabetes: systematic review and meta-analysis. BMJ (Clinical Research Ed.) 2017; 357: j2499. 
https://doi.org/10.1136/bmj.j2499

5. McIntosh C, Demuth H, Pospisilik J, Pederson R. Dipeptidyl peptidase IV inhibitors: How do they work as new antidiabetic agents? Regu Peptides 2005; 128 (2): 15965. https://doi.org/10.1016/j.regpep.2004.06.001

6. Bonora BM, Avogaro A, Fadini GP. Extraglycemic Effects of SGLT2 Inhibitors: A Review of the Evidence. Diabetes, Metabolic Syndrome and Obesity: Targets and Therapy 2020; 13: 161-174.

https://doi.org/10.2147/DMSO.S233538

7. Hemmingsen B, Schroll JB, Wetterslev J, Gluud C, Vaag A, Sonne DP, et al. Sulfonylurea versus metformin monotherapy in patients with type 2 diabetes: a Cochrane systematic review and meta-analysis of randomized clinical trials and trial sequential analysis. CMAJ Open. 2014; 2(3): E162-75. https://doi.org/10.9778/cmajo.20130073

8. Zumla A, Hui DS, Azhar EI, Memish ZA, Maeurer M Reducing mortality from 2019-nCoV: host-directed therapies should be an option. Lancet 2020; 395(10224):e35-e36.

https://doi.org/10.1136/bmi.j2499

9. Zumla A, Chan JF, Azhar EI, Hui DS, Yuen KY. Coronaviruses - drug discovery and therapeutic options. Nat Rev Drug Discov 2016; 15: 327-47. https://doi.org/10.1038/nrd.2015.37

10. Raj VS, Mou H, Smits SL. Dipeptidyl peptidase 4 is a functional receptor for the emerging human coronavirusEMC. Nature 2013;495(7440):251-256. https://doi.org/10.1038/nature12005

11. Iacobellis G. COVID-19 and diabetes: can DPP4 inhibition playarole? Diabetes Res Clin Pract 2020; 162:108125. https://doi.org/10.1016/j.diabres.2020.108125

12. Feng Y, Wang L, Ma X. Effect of hCMSCs and liraglutide combination in ALI through cAMP/PKAc/ $\beta$-catenin signaling pathway. Stem Cell Res Ther 2020; 11(1):2. https://doi.org/10.1186/s13287-019-1492-6 